\title{
“Dao” Cannot Produce “One”
}

\begin{abstract}
Dongkai Li
Independent Scholar

"Dao" refers to the noumenon; "One," “Two," "Three” in the "Dao De Jing” refer to many kinds of independent existence. This paper would argue that the "Dao” cannot produce “One,” and “One” cannot produce “Two.” So, we should realize that the main relation between any independent existence should be the force effected to each other. There are two production ways of the force: One is the external force stated in the study of the physical science; another is from the contradiction within a body, as the demonstration in this paper.
\end{abstract}

Keywords: Dao, noumenon, existence, force, contradiction

“Dao De Jing”- “De Jing” section 42: "Dao produce One, One produce Two, Two produce Three, Three produce everything."

The logic in this sentence has problem.

"Dao produce One," what is the "Dao," what is this "One," how to produce?

"One produce Two," how?

"Two produce Three," how?

"Three produce everything," how?

The logic in the mathematic was one of the reliable logic firstly realized by people in human history. However, above sentence would not be established even with the logic in the mathematics.

This sentence is a kind of figurative description obviously. However, since "Dao De Jing" is a work of philosophy, the logic, but not the literature and art, should be the main deduction way.

There are three questions involved in this sentence: What is the noumenon, what is the specific thing, what is the relation between the noumenon and the specific thing?

This paper is trying to demonstrate in these three aspects to prove that "Dao" cannot produce "One," and “One” cannot produce “Two.”

\section{What Is the Noumenon}

The noumenon refers to the object which is the basis and initial for everything. The basis for everything means that the existence of the noumenon relies on nothing. The initial for everything means that the noumenon is the first in any way, relying on nothing. In this way, we could say that the noumenon is an existence relying on nothing.

"Rely on" means there should be a kind of force affection performing the function between two things at least. "Rely on nothing" means the force needed by an existence comes from this existence only, not from others. Here, we could say that the noumenon should produce force by itself.

Dongkai Li, independent scholar, China; main research field: Ontology, Cognition, and People.

His published philosophy books are What Is the Existence, To Live with Happiness by XUELIN ISBH. 
For an absolute static status, there should be no force. Since the noumenon could produce the force by itself, we should say the static should not be a nature of the noumenon, and the noumenon contains more than one unit within itself.

The force from others means there is a unit receiving the force and other unit delivering the force. For the receiver, the force should come from one unit at least. Then we should say the noumenon should have two aspects within itself, one delivering the force, another one receiving the force.

These two aspects shall never have the possibility to be changed into one single aspect. For this purpose, these two aspects should have a nature of contradiction. Here, we finally come to a conclusion that the noumenon is an existence with a pair of contradiction within its body.

There are many kinds of contradiction. Since the noumenon relies on nothing, the contradiction within the noumenon is not a kind of specific contradiction. This means the contradiction within the noumenon is just a pair of contradiction.

The noumenon relies on nothing, so, there is no material in the noumenon, which means the noumenon is a pure modality of a pair of contradiction.

\section{What Is the Specific Thing}

As per the deduction in part I, an existence containing a pair of contradiction within its body could produce the force sustaining the existence. In this way, such an existence could be independent.

By this way, any object could be distinguished as an independent existence or not. If one object has a pair of contradiction producing the force for itself, its existence could be independent, if not, it is not an independent object, but a kind of accessory belonging to other independent existence.

\section{What Is the Relation between the Noumenon and the Specific Independent Existence}

As per deduction in part I, a pair of contradiction within a body would decide an independent existence. This means that an independent existence contains only one pair of contradiction. From inside the body of such an independent existence, there should be no other independent existence. Here, we come to another conclusion that an independent existence cannot produce another independent existence by itself.

At least two independent existence would produce a new independent existence only in the condition that these two independent existence becomes a pair of contradiction within a same body.

By this sense, whatever the noumenon or any independent existence, it cannot produce a new independent existence by itself.

"Dao" refers to the noumenon, "One," "Two," "Three" in the "Dao De Jing” refer to many kinds of independent existence. By above conclusion, we can see the "Dao" cannot produce "One," and "One” cannot produce "Two."

So, we should realize that the main relation between any independent existence should be the force effected to each other. There are two production ways of the force: one is the external force stated in the study of the physical science, another is from the contradiction within a body, as the demonstration in this paper. 


\section{Works Cited}

Dongkai, Li. What Is the Existence.

Lao, Zi. Dao De Jing. 